\title{
Malignant Ovarian Steroid Cell Tumor
}

National Cancer Institute

\section{Source}

National Cancer Institute. Malignant Ovarian Steroid Cell Tumor. NCI Thesaurus. Code C39981.

A steroid tumor of the ovary which has an aggressive clinical course and metastasizes to other anatomic sites. It is usually of large size and is characterized by nuclear atypia, increased mitotic activity, hemorrhage, and necrosis. Sometimes, patients have abdominal metastases at presentation. 